\title{
Frequency ratio method for seismic modelling of $\gamma$ Doradus stars
}

\section{The role of rotation}

\author{
J. C. Suárez ${ }^{1,2, \star}$, A. Moya ${ }^{2}$, S. Martín-Ruíz ${ }^{1}$, P. J. Amado ${ }^{3}$, A. Grigahcène ${ }^{1}$, and R. Garrido ${ }^{1}$
}

\author{
1 Instituto de Astrofísica de Andalucía (CSIC), CP3004, Granada, Spain \\ e-mail: jcsuarez@iaa.es \\ 2 LESIA, Observatoire de Paris-Meudon, UMR 8109, France \\ 3 ESO Office Santiago, Alonso de Cordova 3107, Santiago 19, Chile
}

Received 22 March 2005 / Accepted 26 July 2005

\section{ABSTRACT}

The effect of rotation on the Frequency Ratio Method (Moya et al. 2005, A\&A, 432, 189) is examined. Its applicability to observed frequencies of rotating $\gamma$ Doradus stars is discussed taking into account the following aspects: the use of a perturbative approach to compute adiabatic oscillation frequencies; the effect of rotation on the observational Brunt-Väisälä integral determination and finally, the problem of disentangling multiplet-like structures from frequency patterns due to the period spacing expected for high-order gravity modes in asymptotic regime. This analysis reveals that the FRM produces reliable results for objects with rotational velocities up to $70 \mathrm{~km} \mathrm{~s}^{-1}$, for which the FRM intrinsic error increases one order of magnitude with respect to the typical FRM errors given in Moya et al. (2005, A\&A, 432, 189). Our computations suggest that, given the spherical degree $\ell$ identification, the FRM may be discriminating for $m=0$ modes, in the sense that the method avoids any misinterpretation induced by the presence of rotationally split multiplet-like structures, which reinforces the robustness of the method. However, if $\ell$ is unknown, such discrimination is not ensured. In order to check the FRM in presence of slow-moderate rotation, we have applied it to the three observed frequencies of the slowly rotating $\left(v \sin i=29 \mathrm{~km} \mathrm{~s}^{-1}\right) \gamma$ Doradus star HD 48501 .

Key words. stars: rotation - stars: oscillations - stars: fundamental parameters - stars: evolution - stars: individual: HD 48501 - stars: general

\section{Introduction}

In Moya et al. (2005, hereafter Paper I), we presented a method for obtaining asteroseismic information of $\gamma$ Doradus stars when at least three oscillation frequencies are observed. This method, from now on called FRM (Frequency Ratio Method), is particularly useful for improving our knowledge on the pulsational behaviour of $\gamma$ Doradus stars through the following aspects: first, the identification of the radial order $n$ and the mode degree $\ell$ of observed frequencies and second, the possibility of constraining models from an additional stellar quantity, J (the integral of the buoyancy or Brunt-Väisälä frequency weighted over the stellar radius along the radiative zone). Such constraints can be interpreted as an increase of the classical observables,

$\mathcal{P}=\mathcal{P}\left(Z, g, L, T_{\mathrm{eff}} ; \frac{f_{\mathrm{o}, i}}{f_{\mathrm{o}, j}}, \mathcal{J}\right)$,

where $Z$ represents the relative metal abundance; $g$ the surface gravity, $L$ the luminosity; $T_{\text {eff }}$ the effective temperature, and finally, $f_{\mathrm{o}, i}$ and $f_{\mathrm{o}, j}$ the observed frequencies. In Paper I,

\footnotetext{
* Associated researcher at (2).
}

the method was applied to the $\gamma$ Doradus star HD 12901, for which a significant reduction of possible representative models of the star was achieved.

The FRM, based on the first-order asymptotic $g$-mode expression given by Tassoul (1980) (see Eq. (1) in Paper I), is built under the assumptions of adiabaticity and non rotation. However, recent spectroscopic measurements of $59 \gamma$ Doradus candidates (Mathias et al. 2004) show rotational velocities ( $v \sin i$ measurements) varying from 10 to $160 \mathrm{~km} \mathrm{~s}^{-1}$. This corresponds to rotational periods around 0.4 to $4-5$ days, in the limit of applicability of a perturbative analysis used here to compute adiabatic oscillation frequencies.

It has been shown by Michel et al. (1999) and references therein, that photometric parameters are sensitive to rotation. In particular, it modifies the determination of the position of the stars in the HR diagram. Since the FRM results are sensitive to such variations, the rotation must thus be included in the set of working parameters

$\mathcal{P}=\mathcal{P}_{\Omega} \leq\left(Z, g, L, T_{\mathrm{eff}} ; \frac{f_{o, i}}{f_{o, j}}, \mathcal{J}, \Omega\right)$

where $\Omega$ represents the rotational velocity of the star. 
Concerning the theoretical adiabatic oscillations, special care must be taken when the oscillation frequencies are of the order of magnitude of the rotational frequency of the star, i.e. when $\sigma_{\Omega} \sim \sigma / 3$. For such cases the perturbative method for computing adiabatic frequencies may start to fail, and other non-perturbative theories should be used. In this context, several works can be found in the literature: Dintrans \& Rieutord (2000), Rieutord \& Dintrans (2002), Dintrans \& Brandenburg (2004), in which non-radial gravity modes of a typical $\gamma$ Doradus star are studied by means of polytropic models.

In addition, the effects of rotation on the oscillation spectra may introduce additional uncertainties to the FRM. The problem arises when the classical $g$-mode frequency pattern may be misidentified with multiplet-like splitting rotationally induced structures. Ideally, when considering complete asymptotic spectra of $\gamma$ Doradus stars, in a first approximation, the asymptotic $g$-mode pattern, whose periods are regularly spaced, can be easily disentangled from multiplet-like structures, which are regularly spaced in frequency. However in practice, unfortunately only a few oscillation modes are observed, and therefore, such direct discrimination is not accomplishable.

The paper is organised as follows: in Sect. 2, the equilibrium models are described. The domain of validity of the FRM when applied to rotating $\gamma$ Doradus stars is analysed in Sect. 3 . In Sect. 4, the problem of multiplet-like structures in the framework of the FRM is discussed. An application of the FRM for the rotating $\gamma$ Doradus star HD 48501 is given in Sect. 5 . Finally, conclusions are summarised in Sect. 6.

\section{The models}

For the purpose of constructing representative models of $\gamma$ Doradus stars the evolutionary code CESAM (Morel 1997) is particularly adapted. Models are built with a precision optimised to compute oscillations, i.e. around 2000 mesh points for the equilibrium model mesh grid, given in the basis of B-splines. To take into account a first order effect of the rotation, equilibrium equations are modified in the manner described in Kippenhahn \& Weigert (1990). In particular, the spherical symmetric contribution of the centrifugal acceleration is included by means of an effective gravity

$g_{\text {eff }}=g-\mathcal{A}_{\mathrm{c}}(r)$,

where $g$ represents the local gravity component, $r$ the radial distance and,

$\mathcal{A}_{\mathrm{c}}(r)=\frac{2}{3} r^{2} \Omega$

the centrifugal acceleration of matter elements at a distance $r$ from the centre of the star. This spherically symmetric contribution of the rotation does not change the shape of the hydrostatic equilibrium equation. Although, the non-spheric components of the centrifugal acceleration are not considered, they are included as a perturbation in the oscillation computation. The total angular momentum of models is assumed to be globally conserved along the evolution of the star.
The physical parametrisation has been adapted for intermediate mass stars (see Paper I). In particular, all equilibrium models used in the present work are computed with: $\alpha_{M L}=$ $l_{\mathrm{m}} / H p=1.8$, for the convective efficiency and $d_{\mathrm{ov}}=l_{\mathrm{ov}} / H p=$ 0.2 for the mixed core overshooting. The $H p$ corresponds to the local pressure scale-height; $l_{\mathrm{m}}$ and $d_{\mathrm{ov}}$ represent the mixing length and the inertial penetration distance of convective bulbs respectively.

\section{The error determination}

The study of the influence of rotation on the FRM constitutes a rather complex task. We aim at identifying possible uncertainties coming from rotation effects and their relevance for the FRM. Such analysis is here focused in three important aspects: first, the regime of application of the perturbative method for obtaining theoretical adiabatic frequencies. Second, the quantification of the error committed when considering the classical analytical expression for asymptotic $g$ modes to study frequency ratios of rotating $\gamma$ Doradus stars. And third, the influence of rotation on the Brunt-Väisälä integrals J .

\subsection{The perturbative approach for adiabatic oscillation frequencies}

The limit for using the perturbative approach to compute adiabatic oscillation frequencies is given by the relation $\sigma / \sigma_{\Omega} \gg 1$, i.e. when the rotational frequency of the star $\left(\sigma_{\Omega}\right)$ is negligible with respect to the the oscillation frequency of modes $(\sigma)$. In the asymptotic regime, $g$ modes are characterised by their low frequency (high radial order $n$ ). According to Dintrans \& Rieutord (2000), the most perturbed modes by rotation are likely those with $\sigma \lesssim 2 \sigma_{\Omega}$, where $2 \sigma_{\Omega}$ corresponds to the Coriolis frequency. Considering the typical rotational periods of $\gamma$ Doradus stars, low frequency gravity modes in asymptotic regime are strongly affected by rotation.

The present analysis is thus restricted to slow and moderately rotating $\gamma$ Doradus stars, that is, those with rotational velocities smaller than $50-70 \mathrm{~km} \mathrm{~s}^{-1}$. Considering representative models with masses in the range of 1.4-1.7 $M_{\odot}$, the limits of the perturbative approach can be established to have $n=[30,40]$ for modes with $\ell \leq 2$, expected to have significant amplitude not smeared out by cancellation effects.

\subsection{The analytic expression for the asymptotic $g$-mode frequencies}

It is convenient to remind that the analytic expression for the asymptotic $g$ modes used in the FRM is obtained under the hypothesis of non-rotation (see Paper I). We attempt here to determine the accuracy of this expression when rotation effects are considered. In this framework, it is conceivable to define an equivalent form of the analytic expression for the asymptotic $g$ modes as:

$\sigma_{n, \ell, \Omega}^{a} \equiv f(n, \ell) \mathcal{J}_{\Omega}$

where the analytic form of $\mathcal{J}_{\Omega}$ integrals remains identical to $\mathcal{J}$ (see Paper I), and whose values are assumed to be different to 

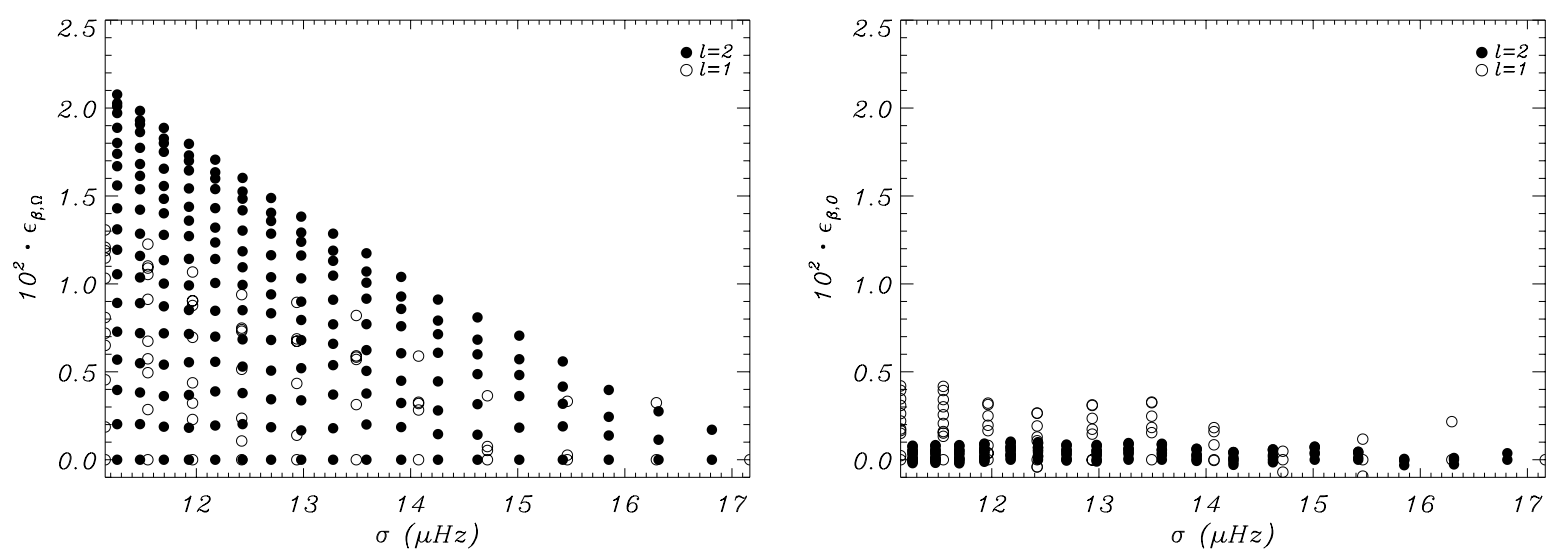

Fig. 1. Relative errors of the asymptotic frequencies as compared to the theoretical predictions for a complete oscillation spectrum obtained from a model with $\Omega \sim 50 \mathrm{~km} \mathrm{~s}^{-1}$. Left panel represent relative errors obtained from oscillations frequency ratios corrected for the effect of rotation. Right panel shows errors without correcting for the effect of rotation.

those obtained without considering rotation effects. The $f(n, \ell)$ functions are a priori unknown and assumed to be only dependent on $\ell$ and $n$ numbers. Similarly as done in Paper I, two given $g$ modes, $\sigma_{\alpha_{1}, \Omega}$ and $\sigma_{\alpha_{2}, \Omega}$, are considered, where $\alpha_{1}$ and $\alpha_{2}$ represent the pairs $\left(n_{1}, \ell_{1}\right)$ and $\left(n_{2}, \ell_{2}\right)$ respectively. Assuming the same mode degree $\ell_{1}=\ell_{2}=\ell$, we make the hypothesis that their eigenfrequencies are approximated by Eq. (1). If the star rotates slowly (cf. Sect. 3.1), $f(n, \ell)$ can be assumed to be:

$f(n, \ell) \sim\left(\frac{\sqrt{\ell(\ell+1)}}{(n+1 / 2) \pi}\right)_{\Omega}$,

and Eq. (1) can thus be rewritten as follows:

$\sigma_{n, \ell, \Omega}^{a}=\left(\frac{\sqrt{\ell(\ell+1)}}{(n+1 / 2) \pi}\right)_{\Omega} \mathcal{J}_{\Omega}$.

In this way, the analytic form proposed by Tassoul (1980) for non-rotating stars is kept and consequently, the formal structure of the FRM remains unaltered. This allows us to relate $\sigma_{\alpha_{1}, \Omega}$ and $\sigma_{\alpha_{2}, \Omega}$ through the following ratio

$\left(\frac{\sigma_{\alpha_{1}}}{\sigma_{\alpha_{2}}}\right)_{\Omega} \approx\left(\frac{n_{2}+1 / 2}{n_{1}+1 / 2}\right)_{\Omega}$.

To obtain this expression, we proceed similarly as in Paper I (Sect. 3). It can be shown that the small differences in the outer turning point location can be considered as negligible as far as the calculation of the Brunt-Väisälä frequency integral is concerned.

Provided theoretical frequencies and the corresponding $n$ 's ratio estimate for a given model, the accuracy of Eq. (4) can be defined as

$\epsilon_{\beta, \Omega}=\left(\frac{\sigma_{i}}{\sigma_{j}}\right)_{\Omega}-\left(\frac{f\left(n_{j}, \ell\right)}{f\left(n_{i}, \ell\right)}\right)_{\Omega}$.

Figure 1 (left panel) shows such errors obtained from a theoretical oscillation spectra of a $1.40 M_{\odot}$ model. It can be noticed that errors reach 0.013 for $\ell=1$ modes, and 0.021 for $\ell=2$ modes, for rotational velocities around $50-60 \mathrm{~km} \mathrm{~s}^{-1}$. These results represent around one order of magnitude larger than the $\epsilon_{\beta, 0}$ errors (right panel), obtained without taking into account corrections for rotation in frequencies. As a consequence, the quantity of valid sets of natural numbers $\left(n_{i}\right)$ increases (see Paper I, Sect. 3). Such increase can be of the order of 10 additional sets for $\ell=1$ modes, and even 100 sets for $\ell=2$ modes. Nevertheless, as explained in Paper I each additional possible set, implies an extra constraint for the Brunt-Väisälä integrals which must be fulfilled.

\subsection{The effects of rotation on the J integrals}

This study must be completed with the analysis of the behaviour of $\mathcal{J}$ integrals when rotation is taken into account.

As explained in Paper I, in the FRM framework the Brunt-Väisälä frequency integrals are calculated in two different manners: from equilibrium models and directly from frequency ratios as deduced from the analytic expression for asymptotic $g$ modes (Eq. (3)). The characteristics of pseudorotating models (cf. Sect. 2) allow us to consider equivalent internal structure for rotating $\gamma$ Doradus stars only modified by an effective gravity. Therefore, the Brunt-Väisälä frequency integrals can be calculated as for non-rotating models, that is

$\mathcal{J}_{\text {th }}=\int_{r_{a}}^{r_{b}} \frac{N}{r} \mathrm{~d} r=\mathcal{J}_{\mathrm{th}, \Omega} \equiv \frac{\sigma_{n, \ell, \Omega}^{a}}{f(n, \ell)}$.

These integrals are dependent mainly on the mass and metallicity of models (Fig. 5 in Paper I). For rotating $\gamma$ Doradus stars it is plausible to consider also a $\Omega$ dependence

$\mathcal{J}_{\text {th, },}=\mathcal{J}_{\text {th }}(M, Z, \Omega)$.

For the purpose of investigating the influence of rotation on Brunt-Väisälä integrals, we consider $1.4 M_{\odot}$ pseudo-rotating models as described in Sect. 2. Models are evolved from the ZAMS to the subgiant region with four different initial rotational velocities: $25,50,100$ and $150 \mathrm{~km} \mathrm{~s}^{-1}$. For these models, the evolution of $\mathcal{J}_{\text {th }, \Omega}$ is depicted in Fig. 2 as a function of the core hydrogen fraction $X_{\mathrm{c}}$. Small variations $(\lesssim 10 \mu \mathrm{Hz})$ are observed. As expected, the effect of considering an 


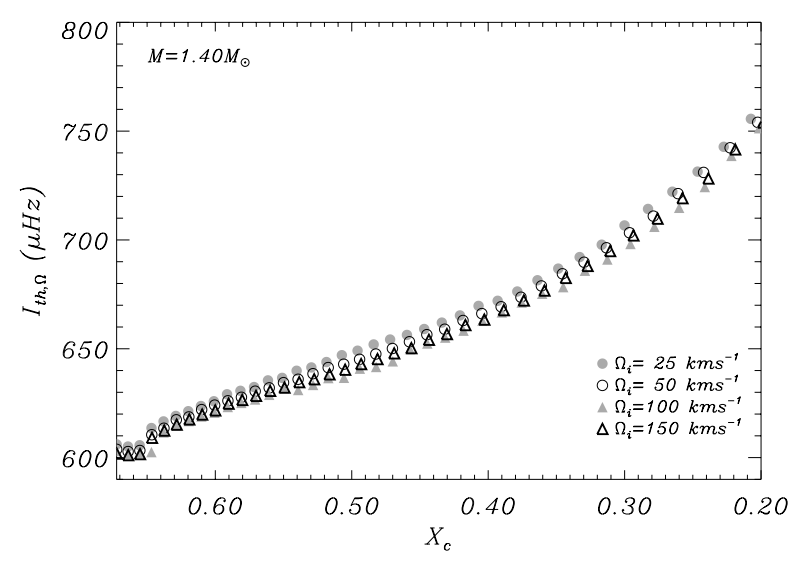

Fig. 2. Variation of theoretical Brunt-Väisälä frequency integral as a function of the evolutionary stage (represented by the central Hydrogen fraction $X_{\mathrm{c}}$ ) for a $1.4 M_{\odot}$ model computed with solar metallicity. Symbols represent models with different initial (ZAMS) rotational velocity.

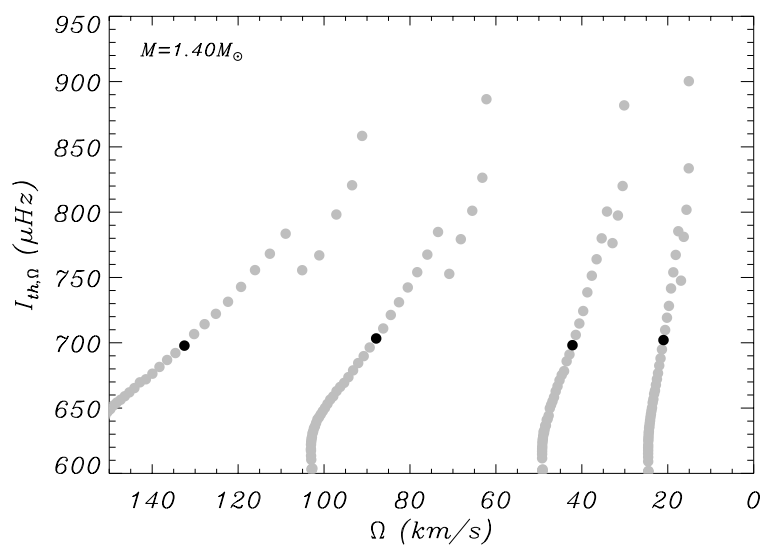

Fig. 3. Theoretical Brunt-Väisälä integral as a function of the rotational velocity $\Omega$. Filled grey circles represent the same $1.4 M_{\odot}$ models evolved with the same initial rotational velocities considered in Fig. 2. Filled black circles represent selected models with the $\mathcal{J}_{\text {th }}$ values closest to $700 \mu \mathrm{Hz}$ (see details in the text).

effective gravity does not modify the behaviour of the theoretical Brunt-Väisälä integrals along the stellar evolution.

In practice, initial rotational velocities are unknown. Therefore, it is worth to analyse the characteristics of models presenting a similar Brunt-Väisälä integral but having been evolved with different initial rotational velocities. In Fig. 3, the evolution of $\mathcal{J}_{\text {th, } \Omega}$ integrals is shown as a function of the rotational velocity. Each track corresponds to evolved models (from left to right) with $\Omega_{\text {ZAMS }}=150,100,50$ and $25 \mathrm{~km} \mathrm{~s}^{-1}$. From each set of models, those with $\mathcal{J}_{\text {th }, \Omega} \sim 700 \mu \mathrm{Hz}$ (black circles) have been arbitrarily selected. The analysis of the corresponding stellar parameters given in Table 1 reveals similar characteristics for the four models. Small differences are mainly due to the different evolutionary stage of models. In particular, the $\pm 5 \mu \mathrm{Hz}$ dispersion in $\mathcal{J}_{\text {th, },}$ values are due to slight differences in their integrands (Fig. 4). As expected, rotation does not discriminate models with respect to their $\mathcal{J}_{\text {th }, \Omega}$. Nevertheless this ensures the coherence of the FRM, since
Table 1. Characteristics of selected models in Fig. 3, presenting a common $\mathcal{J}_{\text {th }} \sim 700 \mu \mathrm{Hz}$, and evolved from the initial (at ZAMS) rotational velocities $25,50,100$ and $150 \mathrm{~km} \mathrm{~s}^{-1}\left(\Omega_{1}, \Omega_{2}, \Omega_{3}\right.$ and $\Omega_{4}$ respectively). The following stellar parameters are given: $T_{\text {eff }}$, the effective temperature (in logarithmic scale); $g$, the surface gravity (in a logarithmic scale); $\bar{\rho}$, the mean stellar density (in $\mathrm{g} \mathrm{cm}^{3}$ ); $R / R_{\odot}$, the stellar radius (in solar units); $X_{\mathrm{c}}$, the central hydrogen fraction; $\Omega$, the rotational velocity (in $\mathrm{km} \mathrm{s}^{-1}$ ), and finally, $\mathcal{J}_{\text {th }}$, the theoretical Brunt-Väisälä frequency integral (in $\mu \mathrm{Hz}$ ).

\begin{tabular}{rrrrr}
\hline \hline & $\Omega_{4}$ & $\Omega_{3}$ & $\Omega_{2}$ & $\Omega_{1}$ \\
\hline $\log T_{\text {eff }}$ & 3.81 & 3.81 & 3.82 & 3.82 \\
$\log g$ & 3.96 & 3.96 & 3.97 & 3.97 \\
$\bar{\rho}$ & 3.79 & 3.75 & 3.88 & 3.84 \\
$R / R_{\odot}$ & 2.18 & 2.19 & 2.17 & 2.17 \\
$X_{\mathrm{c}}$ & 0.32 & 0.30 & 0.29 & 0.28 \\
$\Omega$ & 132.50 & 87.87 & 42.18 & 20.99 \\
$\mathcal{J}_{\text {th }}$ & 697.81 & 703.31 & 698.15 & 702.08 \\
\hline
\end{tabular}

rotation does not introduce additional uncertainties from the point of view of the $\mathcal{J}_{\text {th }}$ behaviour.

Let us consider now the observed Brunt-Väisälä frequency integrals. The analysis of uncertainties of such integrals is not trivial. According to Eq. (1), these integrals can be expressed as

$\mathcal{J}_{\mathrm{obs}, \Omega}=\frac{\sigma_{n, \ell, \Omega}}{f(n, \ell)}$.

From this expression and Eq. (6), it is possible to define the error associated to $\epsilon_{\sigma, \Omega}$ as

$\epsilon_{\sigma, \Omega} \equiv \frac{\left|\sigma_{n, \ell, \Omega}-f(n, \ell) \mathcal{J}_{\mathrm{th}, \Omega}\right|}{\sigma_{n, \ell, \Omega}}$

Respectively, the error associated to the Brunt-Väisälä frequency integral can be written as

$\epsilon_{\mathcal{J}, \Omega} \equiv \frac{\left|\mathcal{J}_{\mathrm{th}, \Omega}-\mathcal{J}_{\mathrm{obs}, \Omega}\right|}{\mathcal{J}_{\mathrm{th}, \Omega}}$

Multiplying both sides of Eq. (10) by $f(n, \ell)^{1}$, and then considering the Eqs. (8) and (9), both errors can be related as follows:

$\frac{\epsilon_{\sigma, \Omega}}{\epsilon_{\mathcal{J}, \Omega}}=\frac{f(n, \ell) \mathcal{J}_{\mathrm{th}, \Omega}}{\sigma_{n, \ell, \Omega}}$

According to Eq. (9), $\epsilon_{\sigma, \Omega}$ can be discussed as a function of the sign of

$1-\frac{f(n, \ell)}{\sigma_{n, \ell, \Omega}} \mathcal{J}_{\mathrm{th}, \Omega}=\left\{\begin{array}{cl}+\epsilon_{\sigma, \Omega} & \text { if } \frac{f(n, \ell)}{\sigma_{n, \ell, \Omega}} \mathcal{J}_{\mathrm{th}, \Omega}>1 \\ -\epsilon_{\sigma, \Omega} & \text { if } \frac{f(n, \ell)}{\sigma_{n, \ell, \Omega}} \mathcal{J}_{\mathrm{th}, \Omega}<1\end{array}\right.$

where the trivial case of $\epsilon_{\sigma, \Omega}=0$ is obviously omitted. The right hand side of Eq. (11) can thus be written as

$\frac{f(n, \ell) \mathcal{J}_{\mathrm{th}, \Omega}}{\sigma_{n, \ell, \Omega}}=1 \pm \epsilon_{n, \ell, \Omega}$

1 This operation is licit since $f(n, \ell)>0$ by construction. 

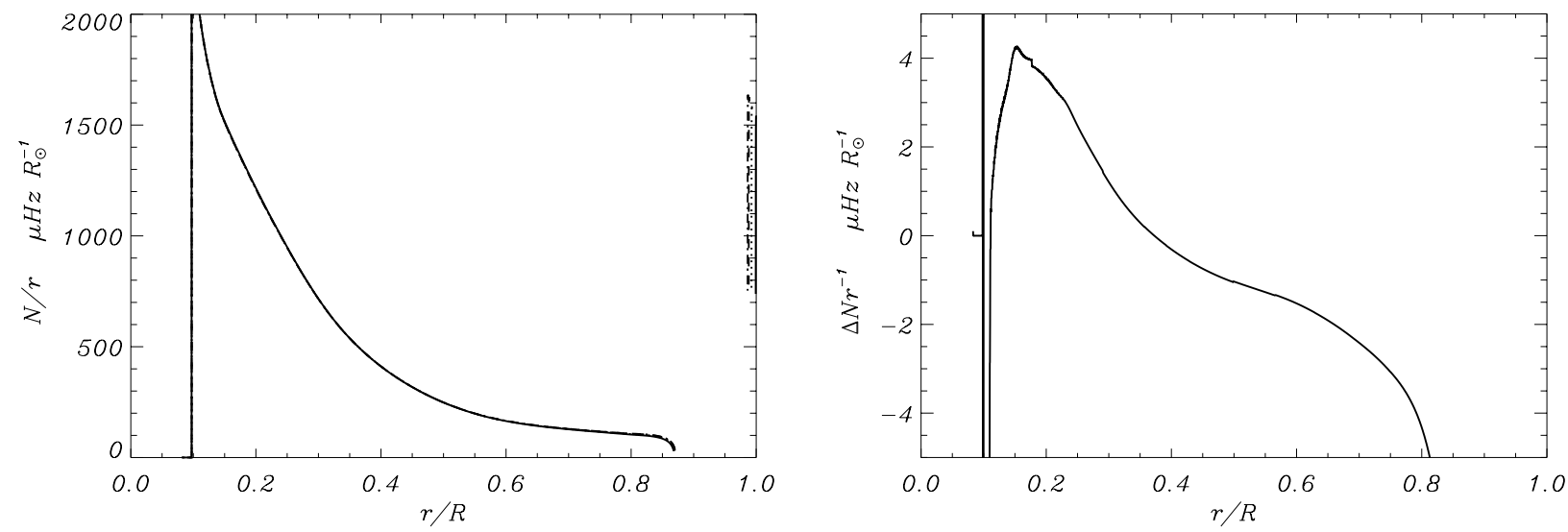

Fig. 4. Brunt-Väisälä frequency weighted over the stellar radius (in solar units) as a function of the radial distance $r$ (normalised to the stellar radius R), for the model with the rotational velocity $\Omega_{1}$ (see Table 1). Right panel shows the relative difference between the Brunt-Väisälä frequency weighted over the stellar radius of models with $\Omega_{1}$ and $\Omega_{4}$ respectively (see Table 1 ).

This allows us to quantify the error in $\mathcal{J}_{\mathrm{obs}, \Omega}$ integrals as a function of $\epsilon_{\sigma, \Omega}$ by replacing the left hand side of Eqs. (11) in (13), yielding

$\epsilon_{\mathcal{J}, \Omega}=\frac{\epsilon_{\sigma, \Omega}}{1 \pm \epsilon_{\sigma, \Omega}}$.

This means that $\epsilon_{\mathcal{J}, \Omega}$ errors can thus be considered of the same order as $\epsilon_{\sigma, \Omega}$. Such result was somehow anticipated in Paper I from numerical computations. Here, although the relation given by Eq. (14) has been obtained for $\sigma_{n, \ell, \Omega}$ and $\mathcal{J}_{\mathrm{obs}, \Omega}$ errors, it can be easily shown that is generally valid whatever considered errors and $f(n, \ell)$ values. Similarly as it happens for non-rotating models, $\epsilon_{\mathfrak{J}, \Omega} \sim 10^{-3}$ which implies an error of $10^{-3}$ in $\epsilon_{\sigma, \Omega}$.

\section{The problem of multiplet-like structures}

When rotational splittings are of the same order as the period spacing of high radial order $g$ modes, i.e.

$\left|\sigma_{n, \ell, m}-\sigma_{n, \ell, m^{\prime}}\right| \sim\left|\sigma_{n, \ell, 0}-\sigma_{n^{\prime}, \ell, 0}\right|$,

the FRM predictions may fail. In particular, rotationally split multiplets (or any of their $m \neq 0$ components) may be confused with the large separation pattern expected for high-order gravity modes in asymptotic regime.

In order to investigate this, an observed oscillation spectrum of a given rotating $\gamma$ Doradus star has been simulated. This simulation has been performed using a pseudo-rotating model (as described in Sect. 2) with a mass of $1.70 M_{\odot}$, and a rotational velocity of $\Omega_{0}=26.8 \mathrm{~km} \mathrm{~s}^{-1}$. Computed with solar metallicity, it is located in the main sequence (age $1.3 \mathrm{Gyr}$ ). It presents an effective temperature and a surface gravity $(\log g$, cgs) of $6918 \mathrm{~K}$ and 3.94 respectively. Its theoretical Brunt-Väisälä frequency is $\mathcal{J}_{\text {th }, \Omega}=667.8 \mu \mathrm{Hz}$.

Adiabatic eigenfrequencies have been computed from this model with the oscillation code FILOU (see Tran Minh \& Léon 1995). In this code, effects of rotation up to second order on the oscillation frequencies have been included (Suárez 2002) following Soufi et al. (1998). Due to the nature of the observed frequencies, the theoretical oscillation computations are extended to high order $g$ modes $(-40 \leq n \leq 1)$, limiting our investigations to modes with degrees $\ell \leq 2$, since cancellation effects are important for the visibility of modes with $\ell \geq 3$.

To cover all the possible situations where multiplet-like structures may contaminate the FRM results, the following scenarios have been explored:

1. the observed frequencies belong to any rotational multiplet;

2 . they belong to modes with different radial order $n$ and azimuthal orders $m$;

3 . they are identified as modes with different $(n)$ but with $m=0$.

\subsection{Scenario 1}

For the first scenario, let us examine, for instance, the observed $(n, \ell)=(22,1)$ triplet with frequencies (in $\mu \mathrm{Hz})$

$\sigma_{-1}=14.756, \sigma_{0}=13.493, \sigma_{+1}=12.045$,

and with frequency ratios

$\frac{\sigma_{+1}}{\sigma_{0}}=0.8927, \frac{\sigma_{0}}{\sigma_{-1}}=0.9144, \frac{\sigma_{+1}}{\sigma_{-1}}=0.8163$.

For these frequency ratios, the FRM predicts a list of possible $(n, \mathcal{J})$ identifications indicated in Table 2 . As discussed in Sect. 3.2, this list has been constructed by assuming an error of $\pm 1.3 \times 10^{-2}$ for $\epsilon_{\beta}$. An estimate of $\mathcal{J}_{\mathrm{obs}, \Omega}$ integrals is also given for the two most relevant mode degree values: $\ell=1$ and 2 . The observed set is obviously not predicted since $n_{1}=n_{2}=n_{3}$. When analysing the list of possible $\mathcal{J}_{\mathrm{obs}, \Omega}$ integrals, predictions exceed the error limit (around 2\%) when assuming $\ell=1$. That is, for this known value of the mode degree, none of the potential sets would be misinterpreted. The same exercise repeated with other $\ell=1$ multiplets within the same spectral range yields similar results. In contrast, the list of Brunt-Väisälä integrals predicted assuming $\ell=2$ contains $\mathcal{J}_{\mathrm{obs}, \Omega}$ values close to the theoretical one. In particular, the set $\left(n_{1}, n_{2}, n_{3}\right)=$ $(43,38,35)$ is related to $\mathcal{J}_{\mathrm{obs}, \Omega}(\ell=2)=666.252 \mu \mathrm{Hz}$, which represents an error of $0.23 \%$ approximately with respect to the $\mathcal{J}_{\text {th }}$. This case would thus be relevant when no additional information on the mode degree is provided. In such a situation, 
Table 2. List of possible $(n, \mathcal{J})$ identifications provided by the FRM applied to the theoretical frequencies belonging to the rotational $(n=$ $22, \ell=1$ ) triplet (more details in text). The first three columns represent the resulting radial order identification. Last two, list the corresponding observed $\mathcal{J}$ when assuming $\ell=1$ and 2 respectively.

\begin{tabular}{rrrrr}
\hline \hline$n_{1}$ & $n_{2}$ & $n_{3}$ & $J_{\text {obs }}(\ell=1)$ & $J_{\text {obs }}(\ell=2)$ \\
\hline 26 & 23 & 21 & 704.379 & 406.673 \\
27 & 24 & 22 & 734.353 & 423.978 \\
28 & 25 & 23 & 764.326 & 441.284 \\
36 & 32 & 29 & 974.141 & 562.421 \\
37 & 33 & 30 & 1004.115 & 579.726 \\
38 & 34 & 31 & 1034.089 & 597.031 \\
39 & 35 & 32 & 1064.062 & 614.336 \\
40 & 36 & 33 & 1094.036 & 631.642 \\
42 & 37 & 34 & 1124.009 & 648.947 \\
43 & 38 & 35 & 1153.983 & 666.252 \\
44 & 39 & 36 & 1183.957 & 683.557 \\
45 & 40 & 37 & 1213.930 & 700.863 \\
\hline
\end{tabular}

the discrimination between a possible multiplet-like structure and asymptotic $g$-mode pattern is not ensured.

\subsection{Scenario 2}

The second scenario implies different radial and azimuthal orders. To check it, the $\ell=1$ modes with $(n, m)=(24,+1)$, $(22,0)$ and $(19,-1)$ have been arbitrarily selected. Their corresponding frequencies are

$\sigma_{19,-1}=16.727, \sigma_{22,0}=13.493, \sigma_{24,+1}=10.979$,

for which the corresponding frequency ratios are

$\frac{\sigma_{+1}}{\sigma_{0}}=0.8137, \frac{\sigma_{0}}{\sigma_{-1}}=0.8066, \frac{\sigma_{+1}}{\sigma_{-1}}=0.6563$.

In this case, the application of the FRM to these frequency ratios yields the list of possible $(n, \mathcal{J})$ identifications given in Table 3. Once again, the solution is not predicted by the FRM. Moreover, the $\mathcal{J}_{\mathrm{obs}, \Omega}$ predictions for $\ell=1$ modes are out of the error limit. However, as discussed in Scenario 1, when considering $\ell=2$ modes, the theoretical Brunt-Väisälä integral is predicted, at least by one of the sets: $\left(n_{1}, n_{2}, n_{3}\right)=(47,38,31)$.

\subsection{Scenario 3}

The third and last scenario is constituted by three centroid modes $(m=0)$, with different radial order. Proceeding as in Scenarios 1 and 2, the frequencies of the $\ell=1$ modes with $n=19,22$ and 24 are

$\sigma_{19}=15,4633, \sigma_{22}=13.4928, \quad \sigma_{24}=12.4232$,

and their frequency ratios are

$\frac{\sigma_{24}}{\sigma_{22}}=0.9207, \frac{\sigma_{22}}{\sigma_{19}}=0.8726, \frac{\sigma_{24}}{\sigma_{19}}=0.8034$.

The $(n, \mathcal{J})$ possible identifications given by the FRM applied to these frequencies are listed in Table 4 . In contrast to the previous scenarios, when considering centroid modes the solution
Table 3. Same as Table 2 but obtained for the case of modes with different radial and azimuthal orders (more details in the text).

\begin{tabular}{rrrrr}
\hline \hline$n_{1}$ & $n_{2}$ & $n_{3}$ & $\mathrm{~J}_{\text {obs }}(\ell=1)$ & $\mathrm{J}_{\text {obs }}(\ell=2)$ \\
\hline 20 & 16 & 13 & 494.564 & 285.536 \\
25 & 20 & 16 & 614.458 & 354.757 \\
26 & 21 & 17 & 644.432 & 372.063 \\
31 & 25 & 20 & 764.326 & 441.284 \\
32 & 26 & 21 & 794.300 & 458.589 \\
37 & 30 & 24 & 914.194 & 527.810 \\
38 & 31 & 25 & 944.168 & 545.115 \\
40 & 32 & 26 & 974.141 & 562.421 \\
42 & 34 & 27 & 1034.089 & 597.031 \\
43 & 35 & 28 & 1064.062 & 614.336 \\
44 & 36 & 29 & 1094.036 & 631.642 \\
45 & 36 & 29 & 1094.036 & 631.642 \\
46 & 37 & 30 & 1124.009 & 648.947 \\
47 & 38 & 31 & 1153.983 & 666.252 \\
48 & 39 & 31 & 1183.957 & 683.557 \\
49 & 40 & 32 & 1213.930 & 700.863 \\
\hline
\end{tabular}

Table 4. Same as Table 2 for the case of modes with different radial order but the same $(m=0)$ azimuthal order (more details in the text).

\begin{tabular}{rrrrr}
\hline \hline$n_{1}$ & $n_{2}$ & $n_{3}$ & $\mathcal{J}_{\text {obs }, \Omega}(\ell=1)$ & $\mathcal{J}_{\text {obs }, \Omega}(\ell=2)$ \\
\hline 24 & 22 & 19 & 674.405 & 389.368 \\
25 & 23 & 20 & 704.379 & 406.673 \\
26 & 24 & 21 & 734.353 & 423.978 \\
34 & 31 & 27 & 944.168 & 545.115 \\
35 & 32 & 28 & 974.141 & 562.421 \\
36 & 33 & 29 & 1004.115 & 579.726 \\
37 & 34 & 30 & 1034.089 & 597.031 \\
39 & 36 & 31 & 1094.036 & 631.642 \\
40 & 37 & 32 & 1124.009 & 648.947 \\
\hline
\end{tabular}

is found by the FRM. Not only the observed radial order set is included in the list of possible solutions (and thereby the mode degree $\ell$ ), but also its related $\mathcal{J}_{\text {obs, } \Omega}$ value predicts the theoretical Brunt-Väisälä integral within the error limits.

\subsection{Considering different rotational velocities}

The previous discussion considers only one rotational velocity. However, usually, it cannot be determined from observations neither the specific surface rotational velocity nor the angle of inclination of the star but the value of $v \sin i$. Since frequency patterns are $\Omega$ dependent, it is plausible to imagine a given rotational velocity for which Eq. (15) holds. To investigate this possibility, 7 models with different rotational velocities have been considered: three models with $\Omega<\Omega_{0}$, three models with $\Omega>\Omega_{0}$ and finally the model used for the theoretical test which rotates at $\Omega_{0}=26.8 \mathrm{~km} \mathrm{~s}^{-1}$. The range of rotational velocities has been chosen as to produce models representative of a typical $\gamma$ Doradus star. The HR diagram error boxes have been obtained from typical multicolour photometry errors of $\gamma$ Doradus stars.

We are looking for any trend indicating that the observed frequencies $\sigma_{19}, \sigma_{22}$, and $\sigma_{24}$ may be misidentified with a 

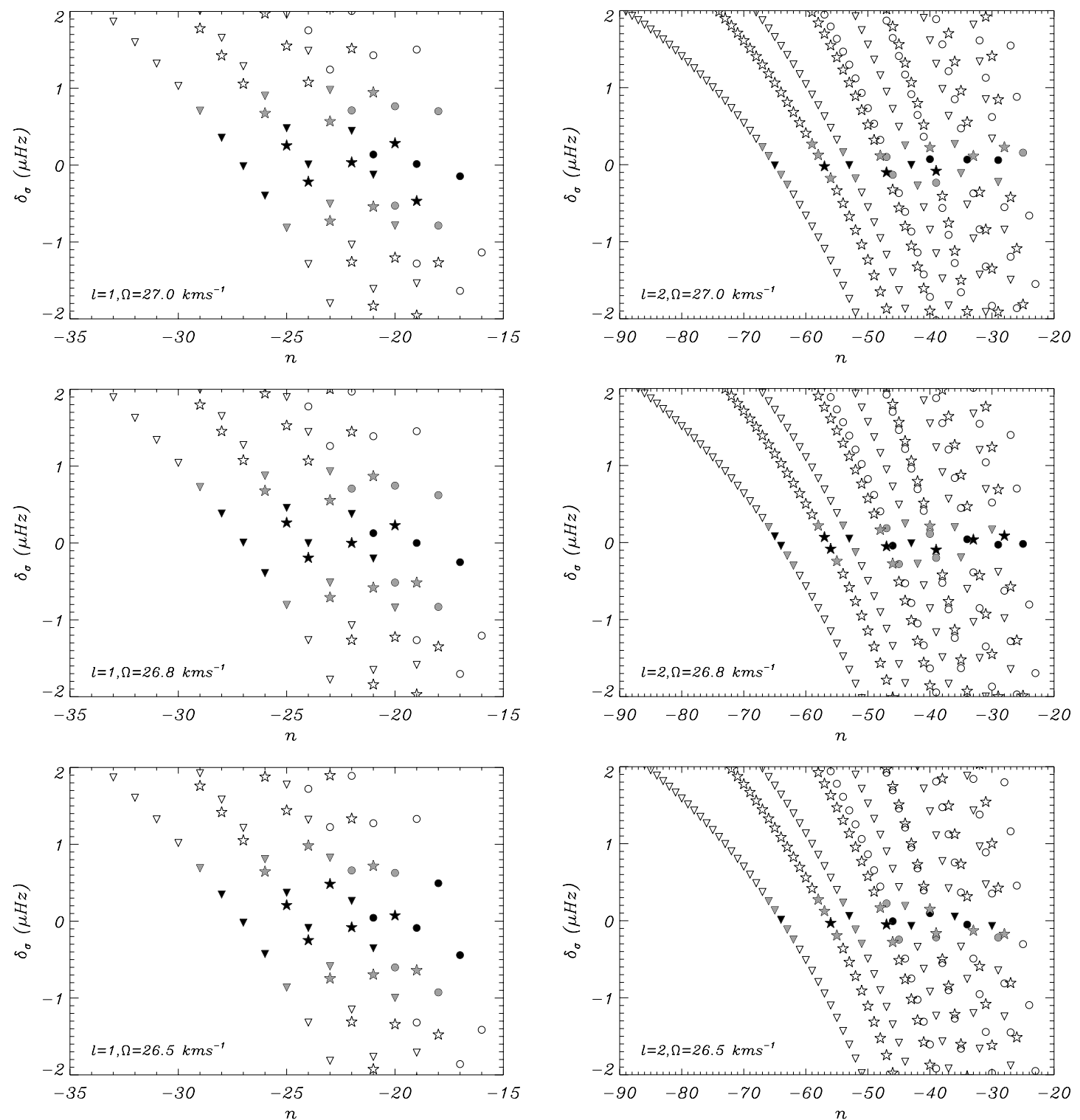

Fig. 5. Frequency differences between the components of the observed modes proposed in Scenario 3 (Sect. 4.3) and those of theoretical multiplets as a function of the radial order $n$. Left and right panels show frequency differences calculated considering $\ell=1$ and $\ell=2$ modes respectively. Symbols represent the frequency differences calculated for the three observed $\ell=1, m=0$ modes: circles for $\sigma_{19}$, triangles for $\sigma_{22}$ and finally, stars for $\sigma_{24}$. Azimuthal orders should be read as going from negative to positive $(m= \pm|\ell|)$ from left to right respectively. Filled circles represent modes for which $\delta_{\sigma} \lesssim 0.5$ (grey) and $\delta_{\sigma} \lesssim 0.1$ (black).

rotational triplet or with components of any $\ell=1,2$ multiplets. To do so, we have calculated the frequency difference $\delta_{\sigma}$ between the observed modes and those of theoretical multiplets for the 7 models considered here. In Fig. 5, such differences are shown for the reference model (central panels), the fastest one (top panels) and the slowest one (bottom panel). In the case of $\ell=1$ triplets (left panels), the smallest $\left|\delta_{\sigma}\right|$ values vary from 0.1 (black filled circles) to 0.5 (grey filled circles) approximately, for a set of 15-20 modes. As expected, for $\ell=2$ modes (right panels) $\delta_{\sigma}$ decreases, and the number of modes with frequencies close to the observed ones increases. In this case, the smallest $\left|\delta_{\sigma}\right|$ values vary from $0.001 \mu \mathrm{Hz}$ (black filled circles) to $0.3 \mu \mathrm{Hz}$ (grey filled circles) approximately, for a set of 35-40 modes. Discarding the exact solution for $\sigma_{19}$, $\sigma_{22}$, and $\sigma_{24}$ for which $\delta_{\sigma}=0$, these frequency differences exceed in at least three orders of magnitude $(\ell=2 \text { results })^{2}$ the observational frequency error typical for $\gamma$ Doradus stars.

In order to consider the smallest differences for the 7 seven models, the minimum $\delta_{\sigma}$ value found for each model is depicted in Fig. 6 as a function of the rotational velocity. Although the mode degree is not here specifically identified, it is found that most of selected modes are $\ell=2$, result somehow expected since, according to Fig. 5, the smallest differences corresponds to $\ell=2$. Obviously, the exact solution $\left(\delta_{\sigma, \min }=0\right.$ at $\Omega=\Omega_{0}$ ) correspond to $\ell=1$ modes. As can be seen, the $\delta_{\sigma, \min }$ distribution does not show any specific trend with the rotational velocity and, quantitatively, the results obtained

${ }^{2}$ For $\ell=1, \delta_{\sigma}$ differences reach five orders of magnitude that of the observational frequency error. 


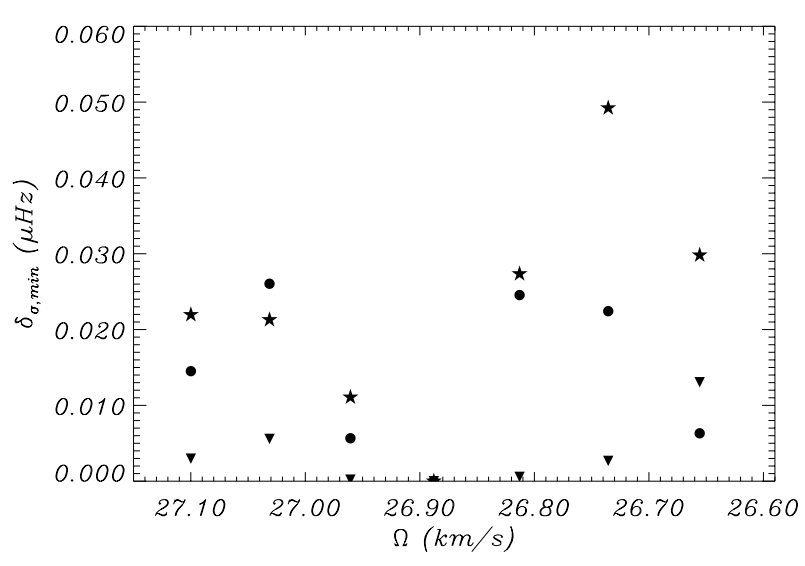

Fig. 6. Variation of the minimum frequency differences considered in Fig. 5 with the rotational velocity. Same symbol representation as in Fig. 5 is adopted.

are similar to those shown in Fig. 5. This result does not exclude the possibility of misidentification, however it shows it is unlikely for the range of models representative of the observed star.

This theoretical test shows that, provided the mode degree $\ell$ is known, the FRM may be discriminating with respect to the multiplet-like structures. In such cases, coherent FRM solutions are restricted to centroid mode $(m=0)$ frequen$\operatorname{cies}^{3}$. When no additional information on $\ell$ is given, the FRM does not discriminate multiplet-like structures. Nevertheless, as shown in this exercise, such a situation is unlikely.

\section{A particular case: the $\gamma$ Doradus star HD 48501}

The $\gamma$ Doradus star HD 48501 is particularly suitable to illustrate the previous theoretical exercise applied to a real star. Having a $v \sin i \sim 30 \mathrm{~km} \mathrm{~s}^{-1}$, it can be considered as a slow rotator, fulfilling thus one of the conditions discussed in Sect. 3. According to a recent frequency analysis reported by Aerts et al. (2004) the star pulsates in at least three modes. In this paper, a discussion has been opened on whether these frequencies could belong to a $\ell=1$ triplet-like structure or to the period spacing expected for high-order gravity modes.

\subsection{Fundamental parameters}

The stellar fundamental parameters of HD 48501 have been determined by applying TempLogG (Kupka \& Bruntt 2001) to the Strömgren-Crawford photometry listed in the Hauck-Mermilliod catalogue (Hauck \& Mermilliod 1998). In this catalogue, no $\beta$ index value is given for HD 48501, which was instead obtained from Handler (1999). The code classifies this object as a main sequence star in the spectral range region F0-G2. The resulting physical parameters are listed in Table 5.

Dupret et al. (2002) gives also physical parameters for HD 48501 based on the Geneva photometry of Aerts et al. (2004) and the calibrations given by Kunzli et al. (1997) for

\footnotetext{
${ }^{3}$ Such result is obviously restricted to stars fulfilling the conditions and errors discussed in Sect. 3.
}

Table 5. Fundamental parameters of HD 48501 taken from the literature. From left to right: effective temperature (in K); surface gravity (cgs, in a logarithmic scale); metallicity and finally the projected rotational velocity (in $\mathrm{km} \mathrm{s}^{-1}$ ). Superindex indicates data were obtained from the following references: ${ }^{a}$ Kupka \& Bruntt (2001); ${ }^{b}$ Dupret et al. (2002); ${ }^{c}$ Cayrel de Strobel et al. (1997); ${ }^{d}$ Aerts et al. (2004) and finally ${ }^{e}$ Royer et al. (2002).

\begin{tabular}{cccc}
\hline \hline $\begin{array}{c}T_{\text {eff }} \\
(\mathrm{K})\end{array}$ & $\begin{array}{c}\log g \\
(\mathrm{dex})\end{array}$ & $\begin{array}{c}{[\mathrm{Fe} / \mathrm{H}]} \\
(\mathrm{dex})\end{array}$ & $\begin{array}{c}v \sin i \\
\left(\mathrm{~km} \mathrm{~s}^{-1}\right)\end{array}$ \\
\hline $\mathbf{6 9 8 4}^{a}$ & $\mathbf{3 . 9 2}^{a}$ & $\mathbf{- 0 . 1 2}^{a}$ & $29^{d}$ \\
$7079^{b}$ & $4.49^{b}$ & $-0.10^{b}$ & $47^{e}$ \\
$7099^{c}$ & $3.96^{c}$ & $+0.01^{c}$ & \\
\hline
\end{tabular}

the Geneva photometry of B to G stars. In his study, theoretical stellar models for these stars do not take into account their surface gravity determinations, for being too high, corresponding to models below the ZAMS. Physical parameters were also retrieved from the fifth edition of the catalogue of $[\mathrm{Fe} / \mathrm{H}]$ determinations by Cayrel de Strobel et al. (1997), which includes $[\mathrm{Fe} / \mathrm{H}]$ and atmospheric parameters $\left(T_{\text {eff }}, \log g\right)$ obtained from high resolution spectroscopic observations and detailed analysis, most of them carried out with the help of model-atmospheres. The aforementioned physical parameters are listed in Table 5.

Values for the $v \sin i$ of were found in Royer et al. (2002), computed from spectra collected at Observatoire de HauteProvence (OHP) and by Aerts et al. (2004), derived from a cross-correlation function analysis of spectroscopic measurements with the CORALIE spectrograph. Very recently, Mathias et al. (2004) have published the results of a two-year long highresolution spectroscopy campaign, monitoring $59 \gamma$ Doradus candidates. In this campaign, more than $60 \%$ of the stars presented line profile variations which can be interpreted as due to pulsation.

\subsection{Locating HD 48501 in the HR diagram}

As usual, the modelling of any star is conditioned by its stellar parameters, delimiting its position in the HR diagram with the corresponding photometric errors. In the present case, the photometric error box (see Fig. 7) has been delimited from fundamental parameters (Table 5) obtained from the StrömgrenCrawford photometry (boldface). For these values, a standard accuracy of 0.2 dex for the surface gravity and $200 \mathrm{~K}$ in $T_{\text {eff }}$ has been considered. In addition, a typical correction for the effect of rotation is included as well. For main sequence intermediate mass stars, this represents $\pm 200 \mathrm{~K}$ approximately (details in Michel et al. 1999; Pérez Hernández et al. 1999), and more recently in Suárez et al. (2002). In the same figure, several evolutionary tracks are drawn to illustrate the wide range of different models that can be considered as representative of HD 48501. In particular, the location in the HR diagram of evolutionary tracks can be modified significantly using different rotational velocities. This becomes critical when different values for the rotational velocity and the metallicity are taken into account. At present, there is no additional information constraining the 
angle of inclination of HD 48501. Therefore, a wide range of rotational velocities should be considered. However, in the present case, in order to respect the hypothesis of applicability of the FRM, we restrict our modelling to low rotational velocities. In Fig. 7, the upper continuous line and the lower dashed line represent, respectively, the evolutionary tracks of a $1.76 M_{\odot}$ non rotating star model and a $1.65 M_{\odot}$ pseudo-rotating star model. These tracks illustrate somehow the limits for the mass and rotational velocities predicted by the modelling.

Four different masses have been considered: from bottom to top, $1.65 M_{\odot}$ ( $D$ models), $1.70 M_{\odot}\left(B\right.$ models) and $1.75 M_{\odot}$ (A models) computed with solar metallicity. To cover the observed $v \sin i$ found in the literature (see Table 5), the following rotational velocities have been used: $30 \mathrm{~km} \mathrm{~s}^{-1}\left(A_{1}, B_{1}\right)$ and $50 \mathrm{~km} \mathrm{~s}^{-1}\left(A_{2}, B_{2}\right)$, corresponding to an angle of inclination of $90^{\circ}$ and $37^{\circ}$ respectively.

\subsection{Applying the FRM to the observed frequencies}

The three observed frequencies (Table 6) of HD 48501 have been taken from Aerts et al. (2004). The corresponding frequency ratios are

$\frac{f_{\mathrm{I}}}{f_{\text {II }}}=0.9123, \frac{f_{\mathrm{II}}}{f_{\text {III }}}=0.9292, \frac{f_{\mathrm{I}}}{f_{\text {III }}}=0.8478$.

All possible natural number ratios up to $n=60$ are then calculated. As described in Sect. 3, we assume an error of $\pm 1.3 \times 10^{-2}$ for the calculation of possible $n$ sets, instead of the standard error of $\pm 5 \times 10^{-3}$ used under the assumption of non-rotation (cf. Paper I). The results are detailed in Table 7 , for which only dipoles $(\ell=1)$ and quadrupoles $(\ell=2)$ have been considered up to $n \lesssim 50$.

Within the aforementioned errors, seven possible $n$ sets are obtained. Although, most of them (5 of 7) are predicted as $\ell=2$ modes, the $t_{4}$ and $t_{6}$ are predicted as $\ell=1$ modes. This latter possibility would thus support the results obtained with multicolour photometry given by Aerts et al. (2004). The corresponding $\mathcal{J}_{\text {obs }, \Omega}$ integrals (see Table 7) are compared with theoretical predictions in the $\mathrm{J}_{\text {th }}-T_{\text {eff }}$ diagram given in Fig. 8 . When analysing such comparison for $\ell=1$ possibilities (shaded boxes), no successful results are afforded, i.e. the models fulfilling the $\left(n_{i}, \mathcal{J}\right)$ predictions are not consistent with the observational constraints. In particular, the observed metallicity and the corresponding $\mathcal{J}_{\mathrm{th}, \Omega}$ predictions are in clear dissonance. In particular, the FRM would predict models with a metallicity in the range of $[\mathrm{Fe} / \mathrm{H}]=[-0.4,-0.6]$. In contrast, when considering possible sets with $\ell=2$, the FRM predicts (according to the $\mathcal{J}_{\text {obs }, \Omega}$ integrals) models with the observed metallicity $[\mathrm{Fe} / \mathrm{H}] \geq 0.1$, in particular for $t_{1}, t_{2}$ and $t_{3}$ sets. Such solutions would correspond to models with masses from 1.4 to $1.6 M_{\odot}$ in the main sequence.

\subsection{The observed frequencies as belonging to a rotational split $\ell=1$ triplet}

The previous results suggest, if $\ell=1$ confirmed, the alternative of considering the observed frequencies as belonging to one or more multiplet-like structure split by rotation.
Table 6. Photometric data for the $\gamma$ Doradus star HD 48501. Observed frequencies, in both cycles per day and $\mu \mathrm{Hz}$, are taken from Aerts et al. (2004).

\begin{tabular}{ccc}
\hline \hline & $(c / d)$ & $(\mu \mathrm{Hz})$ \\
\hline$f_{\mathrm{I}}$ & 1.095 & 12.663 \\
$f_{\mathrm{II}}$ & 1.199 & 13.880 \\
$f_{\text {III }}$ & 1.290 & 14.937 \\
\hline
\end{tabular}

Table 7. List of selected natural numbers sets associated to the observed frequency ratios of HD 48501. For each set, the corresponding observed $\mathcal{J}_{\text {obs }, \Omega}$ is given in $\mu \mathrm{Hz}$. Finally, an estimate of the spherical order $\ell$ is also given in Col. 4 .

\begin{tabular}{llllll}
\hline \hline & $n_{1}$ & $n_{2}$ & $n_{3}$ & $\ell$ & $\mathcal{J}_{\text {obs }}$ \\
\hline$t_{1}$ & 38 & 41 & 45 & 2 & 739 \\
$t_{2}$ & 39 & 42 & 46 & 2 & 757 \\
$t_{3}$ & 40 & 43 & 47 & 2 & 776 \\
$t_{4}$ & 27 & 29 & 32 & 1 & 912 \\
$t_{5}$ & 27 & 29 & 32 & 2 & 527 \\
$t_{6}$ & 28 & 30 & 33 & 1 & 946 \\
$t_{7}$ & 28 & 30 & 33 & 2 & 546 \\
\hline
\end{tabular}

In Fig. 9, the theoretical evolution of adiabatic frequencies is given for two representative models of HD 48501. The region where the observed frequencies (continuous horizontal lines) can be identified is around the triplet $g_{22}$, that is a $n=22$, $g$ mode. This figure shows that theoretical predictions fit better for higher masses (right panel) and for evolved models (lower effective temperatures).

Two rotational velocities are considered: $30 \mathrm{~km} \mathrm{~s}^{-1}$, and $50 \mathrm{~km} \mathrm{~s}^{-1}$, represented by dotted and dashed lines respectively. For the low-mass model (Fig. 9, left panel), the predicted triplet structure is clearly shifted with respect to observed frequencies for both rotational velocities. In the case of the $1.70 M_{\odot}$ model, the predicted $m=0$ frequency obtained when considering $\Omega=50 \mathrm{~km} \mathrm{~s}^{-1}$ is centred with respect to the observed ones. However the $m= \pm 1$ branches are still far from the observations. In contrast, when considering the $\Omega=30 \mathrm{~km} \mathrm{~s}^{-1}$ rotational velocity, theoretical frequencies tend to the observed ones for the low effective temperatures. Such results would thus suggest that $1.70 M_{\odot}$ pseudo-rotating models, with effective temperatures near the lower limit of the error box, and with a rotation velocity around $30 \mathrm{~km} \mathrm{~s}^{-1}$ may be considered as representative for HD 48501. In addition, the rotational velocity confirms the spectroscopic $v \sin i$ observation.

In order to confirm the triplet possibility, it is worth to analyse the behaviour of the average splitting predicted by models. For the rotational velocities considered here, the frequency structure of a rotationally split $\ell=1$ can be studied by using the mean splitting form

$\Delta_{\mathrm{s}} \equiv \frac{1}{2}\left(\sigma_{+1}-\sigma_{-1}\right)$.

In Fig. 10 (left panel) the evolution of the theoretical splitting $\left(\ell=1, g_{22}\right)$ is shown for the model giving the best frequency results $\left(1.70 M_{\odot}\right)$. The solid line represents the observed average spacing, which value is $\Delta_{\mathrm{s}}^{\mathrm{obs}}=1.14 \mu \mathrm{Hz}$. When 


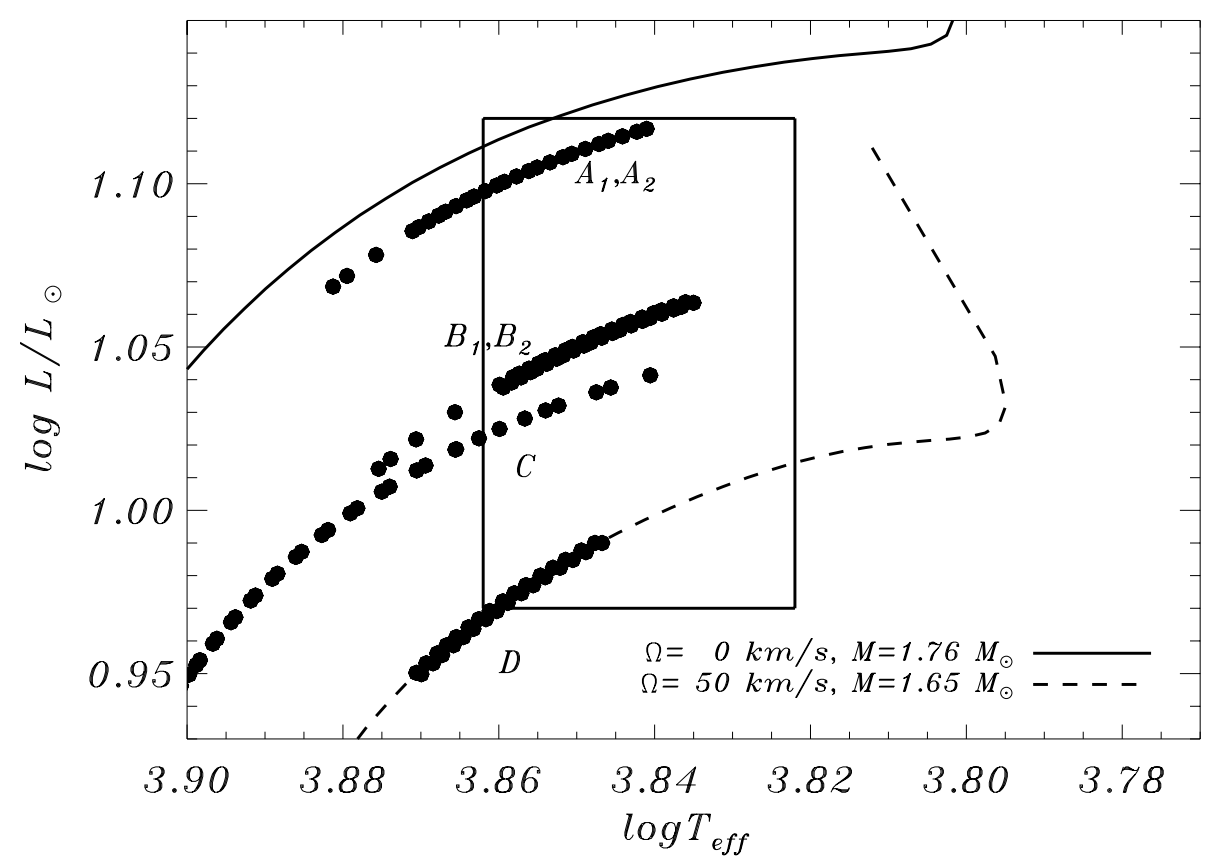

Fig. 7. HR diagram containing evolutionary tracks and models loci considered in this work. The continuous line represents the evolutionary track of a $1.76 M_{\odot}$ non rotating star model. Respectively, the dashed line represents an evolutionary track of an $1.65 M_{\odot}$ pseudo-rotating star model, considering a rotational velocity of $50 \mathrm{~km} \mathrm{~s}^{-1}$. $A, B$ and $D$ correspond to the location of $1.75 M_{\odot}, 1.70 M_{\odot}$ and $1.65 M_{\odot}$ pseudo-rotating models respectively (a solar metallicity is considered). Subindexes 1 and 2 represent values of rotation velocities at the stellar surface of $30 \mathrm{~km} \mathrm{~s}^{-1}$ and $50 \mathrm{~km} \mathrm{~s}^{-1}$ respectively. $C$ represents models of $1.50 M_{\odot}$, with a metallicity of $Z=0.07$ and a rotational velocity of $50 \mathrm{~km} \mathrm{~s}{ }^{-1}$. Models are displayed with filled circles. Finally, the error box considered for HD 48501 is also depicted.

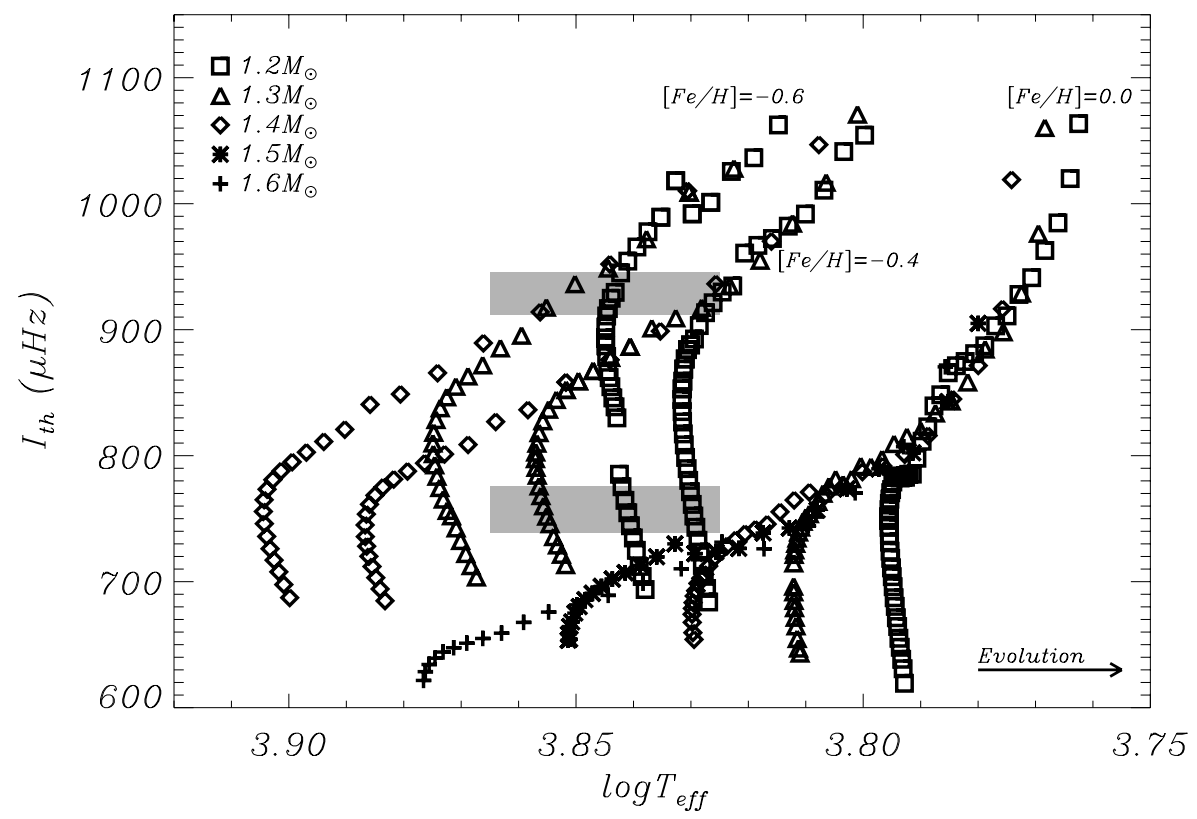

Fig. 8. Theoretical Brunt-Väisälä frequency integral as a function of the effective temperature for representative models of HD 48501 with masses in the range of $M=1.2-1.4 M_{\odot}$, for $[\mathrm{Fe} / \mathrm{H}]=-0.4$ and -0.6 , and $M=1.2-1.6 M_{\odot}$ for $[\mathrm{Fe} / \mathrm{H}]=0.0,-0.4$ and -0.6 . Shaded areas represent uncertainty $\left(\mathcal{J}_{\mathrm{obs}}, T_{\text {eff }}\right)$ boxes of sets $t_{1}-t_{3}$ and $t_{4}, t_{6}$ as given in Table 7 .

considering a rotational velocity of $30 \mathrm{~km} \mathrm{~s}^{-1}$ (dotted line), the observed mean splitting is reproduced $\left(\Delta_{\mathrm{s}}^{\text {th }}-\Delta_{\mathrm{s}}^{\mathrm{obs}} \sim 10^{-3}-10^{-2}\right)$ by models in the region of low temperatures.

In addition, the study of the observed asymmetry may furnish additional information to identify the corresponding triplet. An equivalent form to Eq. (16) can be used to analyse the corresponding $\ell=1$ triplet asymmetry:

$\Delta_{\mathrm{A}} \equiv \sigma_{+1}+\sigma_{-1}-2 \sigma_{0}$,

where the subscripts $+1,-1$ and 0 indicate the corresponding values of the azimuthal order $m$. The evolution of theoretical $\Delta_{\mathrm{A}}$ values as obtained from models rotating with $50 \mathrm{~km} \mathrm{~s}^{-1}$ 

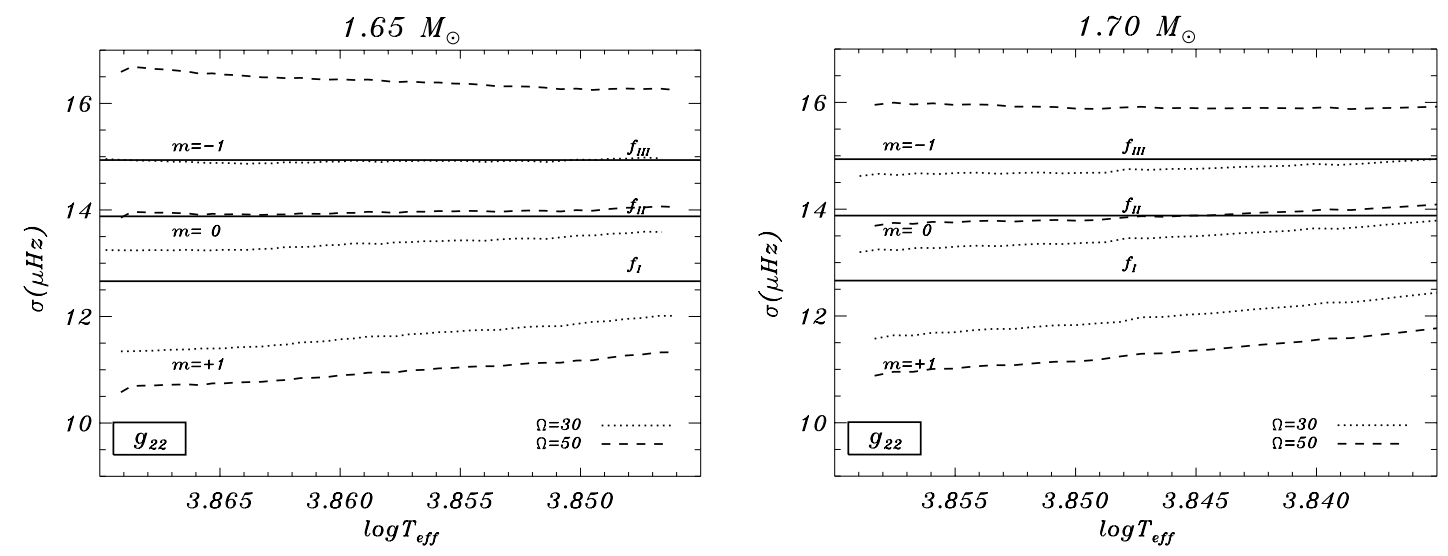

Fig. 9. Evolution of adiabatic oscillation frequencies for the $1.65 M_{\odot}$ and $1.70 M_{\odot}$ models within the HD 48501 photometric error box (left and right panels respectively). Only the three components of the $g_{22}$ triplet are displayed. Dotted lines represent the frequencies obtained from models with a rotational velocity of $30 \mathrm{~km} \mathrm{~s}^{-1}$, and dashed lines correspond to those obtained from models with a rotational velocity of $50 \mathrm{~km} \mathrm{~s}^{-1}$. Finally, the observed frequencies are represented by continuous horizontal lines.
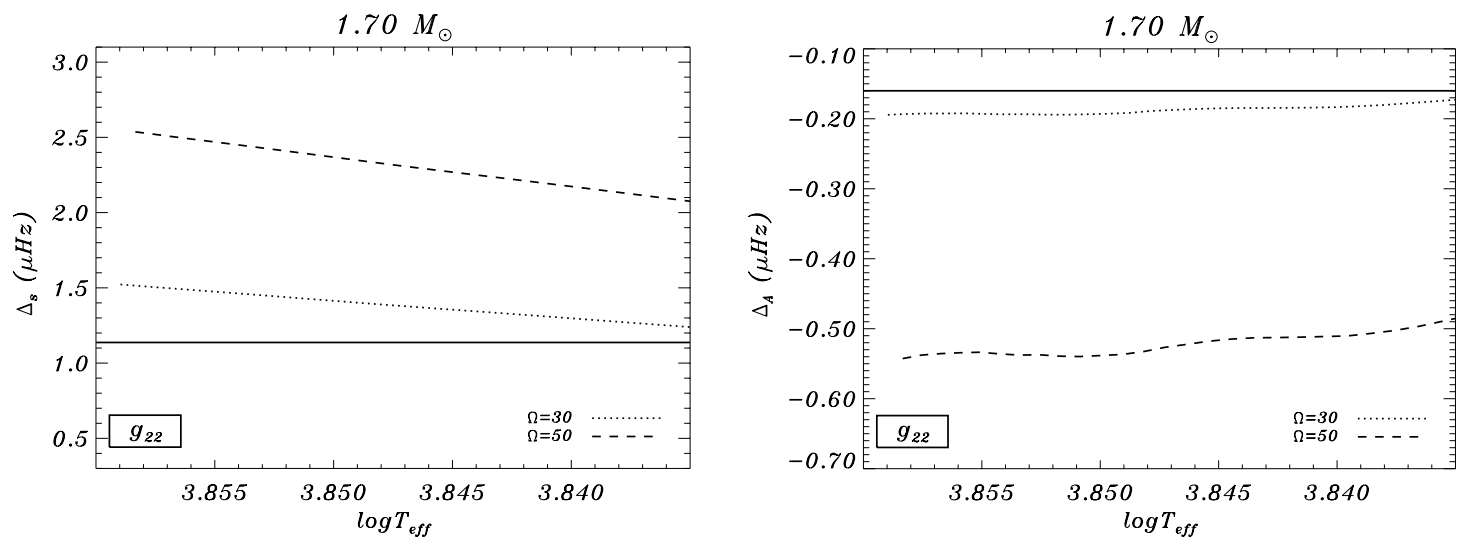

Fig. 10. Mean splitting (left panel) and asymmetry (right panel) of the selected $(\ell, n)=(1,22)$ triplet of the $1.70 M_{\odot}$ models as calculated in Eqs. (16) and (17) respectively. Solid lines represent observed values. Dotted and dashed lines represent theoretical values obtained from models with a rotational velocity of 30 and $50 \mathrm{~km} \mathrm{~s}^{-1}$ respectively.

and $30 \mathrm{~km} \mathrm{~s}^{-1}$ are compared with the observed one $\Delta_{\mathrm{A}}^{\mathrm{obs}}=$ -0.16 in Fig. 10 (right panel). Our best predicted asymmetry is obtained for models with rotational velocities of $30 \mathrm{~km} \mathrm{~s}^{-1}$. In the region of low temperatures these models show $\Delta_{\text {th }}^{\text {obs }}$ approaching to the observed $\Delta_{\mathrm{A}}^{\text {obs }}$ value in approximately $10^{-2}$ orders of magnitude.

The best results for $\Delta_{\mathrm{A}}$ and $\Delta_{\mathrm{S}}$ are given for a $1.70 M_{\odot}$ model with a rotational velocity of $\Omega=28.3 \mathrm{~km} \mathrm{~s}^{-1}$, a radius of $2.43 R_{\odot}$, and presenting an effective temperature of $6839.24 \mathrm{~K}$ $\left(\log T_{\text {eff }}=3.835\right)$ and $\log g=3.896$. Its central hydrogen fraction, $X_{\mathrm{c}}=0.26$, shows an evolutionary stage corresponding to a main sequence star with $1.4 \mathrm{Gyr}$.

\section{Conclusions}

In the present work, the Frequency Ratio Method (FRM) proposed by Moya et al. (2005) is revisited in terms of the applicability to rotating $\gamma$ Doradus stars. The accuracy of the FRM in presence of rotation has been exhaustively examined. To do so, the following aspects have been considered: the validity of a perturbative approach to compute adiabatic oscillation frequencies of $g$ modes in asymptotic regime; the effect of rotation on the observational Brunt-Väisälä frequency integral and, finally, the problem of multiplet-like structures. This problem concerns the possibility of disentangling whether the observed frequencies belong to any rotational split multiplet or to the period spacing expected for high-order gravity modes in asymptotic regime.

It is found that reliable results may be obtained when objects rotate with $\Omega \lesssim 70 \mathrm{~km} \mathrm{~s}^{-1}$. In such cases, the error of the FRM increases on order of magnitude respect to the typical errors given in Paper I.

Concerning the multiplet-like structures, all the possible confusing scenarios have been investigated. Provided any additional information on the mode degree $\ell$, simulations indicate that the FRM would be discriminating for $m=0$ modes. For such cases, any misinterpretation induced by the presence of rotationally split multiplet-like structures is avoided. When $\ell$ is a priori unknown, such discrimination is not ensured. Nevertheless, we show here that the possibility of a confusing scenario is rather unlikely. This constitutes a very important result, since it reinforces and extends (up to some extent) the applicability of the FRM to slowly-moderately rotating $\gamma$ Doradus stars. 
In order to test the present results with a real star the FRM is applied to the $\gamma$ Doradus star HD 48501, which can be considered as a slow rotator $\left(v \sin i=29 \mathrm{~km} \mathrm{~s}^{-1}\right)$. The models fulfilling the corresponding $\left(n_{i}, \mathcal{J}\right)$ predictions were found to be inconsistent with the observational constraints for $\ell=1$ modes, in particular with the observed metallicity. However, for $\ell=2$ modes, the FRM predicts models with masses from 1.4 to $1.6 M_{\odot}$ and radial order ranges in the range $n=[38,47]$.

Furthermore, in contrast to predictions given by Aerts et al. (2004), when analysing the observed frequencies as belonging to a rotational induced $\ell=1$ triplet, solutions reproducing the observed average splitting and the triplet average asymmetry have been found to be plausible for a $1.70 M_{\odot}$ model.

Acknowledgements. This study would not have been possible without the financial support from the PNAYA (National Plan of Astronomy and Astrophysics, Spain), with the project AYA2003-04651. As well, this project was also partially financed by the Spanish "Consejería de Innovación, Ciencia y Empresa" from the "Junta de Andalucía" local government, and by the Spanish Plan Nacional del Espacio under project ESP2004-03855-C03-01. SMR acknowledges financial support by an "Averroes" postdoctoral contract, from the Junta de Andalucía local government.

\section{References}

Aerts, C., Cuypers, J., De Cat, P., et al. 2004, A\&A, 415, 1079

Cayrel de Strobel, G., Soubiran, C., Friel, E. D., Ralite, N., \& Francois, P. 1997, A\&AS, 124, 299
Dintrans, B., \& Brandenburg, A. 2004, A\&A, 421, 775

Dintrans, B., \& Rieutord, M. 2000, A\&A, 354, 86

Dupret, M.-A., De Ridder, J., Neuforge, C., Aerts, C., \& Scuflaire, R. 2002, A\&A, 385, 563

Handler, G. 1999, MNRAS, 309, L19

Hauck, B., \& Mermilliod, M. 1998, A\&AS, 129, 431

Kippenhahn, R., \& Weigert, A. 1990, Stellar structure and evolution, Astronomy and Astrophysics library (Springer-Verlag)

Kunzli, M., North, P., Kurucz, R. L., \& Nicolet, B. 1997, A\&AS, 122, 51

Kupka, F., \& Bruntt, H. 2001, in First COROT/MONS/MOST Ground Support Workshop, 39

Mathias, P., Le Contel, J.-M., Chapellier, E., et al. 2004, A\&A, 417, 189

Michel, E., Hernández, M. M., Houdek, G., et al. 1999, A\&A, 342, 153

Morel, P. 1997, A\&AS, 124, 597

Moya, A., Suárez, J. C., Amado, P. J., Martín-Ruíz, S., \& Garrido, R. 2005, A\&A, 432, 189

Pérez Hernández, F., Claret, A., Hernández, M. M., \& Michel, E. 1999, A\&A, 346, 586

Rieutord, M., \& Dintrans, B. 2002, MNRAS, 337, 1087

Royer, F., Grenier, S., Baylac, M.-O., Gómez, A. E., \& Zorec, J. 2002, A\&A, 393, 897

Soufi, F., Goupil, M. J., \& Dziembowski, W. A. 1998, A\&A, 334, 911 Suárez, J. C. 2002, Ph.D. Thesis

Suárez, J.-C., Michel, E., Pérez Hernández, F., et al. 2002, A\&A, 390, 523

Tassoul, M. 1980, ApJS, 43, 469

Tran Minh, F., \& Léon, L. 1995, Physical Process in Astrophysics, 219 